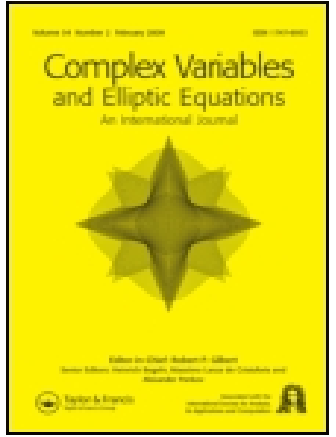

Complex Variables and Elliptic Equations

An International Journal

ISSN: 1747-6933 (Print) 1747-6941 (Online) Journal homepage: http://www.tandfonline.com/loi/gcov20

\title{
Quaternionic Hilbert spaces and a von Neumann inequality
}

\section{Daniel Alpay \& H. Turgay Kaptanoğlu}

To cite this article: Daniel Alpay \& H. Turgay Kaptanoğlu (2012) Quaternionic Hilbert spaces and a von Neumann inequality, Complex Variables and Elliptic Equations, 57:6, 667-675, DOI: 10.1080/17476933.2010.534141

To link to this article: $\underline{h t t p: / / d x . d o i . o r g / 10.1080 / 17476933.2010 .534141 ~}$

曲 Published online: 16 Mar 2011.

Submit your article to this journal ๘

Џlll Article views: 100

Q View related articles $\asymp$

4 Citing articles: 2 View citing articles 


\title{
Quaternionic Hilbert spaces and a von Neumann inequality
}

\author{
Daniel Alpay $^{\text {a* }}$ and H. Turgay Kaptanoğlu ${ }^{\mathrm{b}}$ \\ ${ }^{a}$ Department of Mathematics, Ben-Gurion University of the Negev, P.O. Box 653, \\ Beer-Sheva 84105, Israel; ${ }^{b}$ Department of Mathematics, \\ Bilkent University, Ankara 06800, Turkey
}

Communicated by M. Lanza de Cristoforis

(Received 12 July 2008; final version received 9 August 2010)

\begin{abstract}
We show that Drury's proof of the generalisation of the von Neumann inequality to the case of contractive rows of $N$-tuples of commuting operators still holds in the quaternionic case. The arguments require a seemingly new result on tensor products of quaternionic Hilbert spaces.
\end{abstract}

Keywords: von Neumann inequality; Drury-Arveson space; quaternionic Hilbert spaces; reproducing kernel Hilbert spaces; tensor products

AMS Subject Classifications: Primary 47A60; Secondary 46A32; 47B32; $47 \mathrm{~S} 10$

\section{Introduction}

In 1978, Drury extended von Neumann inequality to the case of contractive rows of $\mathrm{N}$-tuples of commuting operators [1]. Such an extension was done by Arveson as well in [2, Theorem 8.1]; see also [3]. To state their result, we first consider the reproducing kernel Hilbert space $\mathcal{A}$ with reproducing kernel $(1-\langle z, w\rangle)^{-1}$, where $z$ and $w$ belong to the unit ball $\mathbb{B}_{N}$ of $\mathbb{C}^{N}$ and $\langle\cdot, \cdot\rangle$ denotes the inner product of $\mathbb{C}^{N}$. This space is often called the Drury-Arveson space. Letting $\omega_{\alpha}=|\alpha| ! / \alpha$ !, it can also be described as

$$
\mathcal{A}=\left\{f(z)=\sum_{\alpha \in \mathbb{N}^{N}} z^{\alpha} f_{\alpha}:\|f\|_{\mathcal{A}}^{2}:=\sum_{\alpha \in \mathbb{N}^{N}} \frac{\left|f_{\alpha}\right|^{2}}{\omega_{\alpha}}<\infty\right\} .
$$

We have used above the usual multi-index notation in which $\alpha !=\alpha_{1} ! \cdots \alpha_{N} !$ and $|\alpha|=\alpha_{1}+\cdots+\alpha_{N}$ for $\alpha=\left(\alpha_{1}, \ldots, \alpha_{N}\right) \in \mathbb{N}^{N}$. Further, for two multi-indices $\alpha$ and $\beta$, we write $\alpha \geq \beta$ if $\alpha_{\ell} \geq \beta_{\ell}$ for all $\ell=1, \ldots, N$.

Next let $e^{\ell}$ denote the $N$-row vector with all entries 0 with the exception of the $\ell$-th which is 1 , and let the backward shift operators $R_{\ell}$ be defined by

$$
R_{\ell} f(z)=\sum_{\alpha \in \mathbb{N}^{N}} z^{\alpha-e^{\ell}} \frac{\alpha_{\ell}}{|\alpha|} f_{\alpha}
$$

*Corresponding author. Email: dany@math.bgu.ac.il

ISSN 1747-6933 print/ISSN 1747-6941 online 
for $\ell=1, \ldots, N$; if $\alpha_{\ell}=0$, we set $z^{\alpha-e^{\ell}} \frac{\alpha_{\ell}}{|\alpha|}=0$ in the above sum. These operators are bounded on $\mathcal{A}$ and mutually commute. Moreover,

$$
\left(R_{\ell}^{*} f\right)(z)=z_{\ell} f(z)=:\left(M_{\ell} f\right)(z)
$$

which define the forward shift operators $M_{\ell}$, and

$$
\sum_{\ell=1}^{N} R_{\ell}^{*} R_{\ell}=I_{\mathcal{A}}-C C^{*} \leq I_{\mathcal{A}}, \quad \text { where } \quad C f=f(0),
$$

$I$ is the identity operator, and for operators $A, B$ on a Hilbert space $\mathcal{H}$, the equivalent expressions $A \leq B$ and $B-A \geq 0$ denote that $B-A$ is positive in the sense that $\langle(B-A) h, h\rangle_{\mathcal{H}} \geq 0$ for all $h \in \mathcal{H}$ (see [2] for a proof of these facts). They can also be found in a number of later publications (see, e.g. [4]). We can now state the Drury-Arveson result.

THEOREM 1.1 Let $A_{1}, \ldots, A_{N}$ be bounded mutually commuting operators on a Hilbert space $\mathcal{H}$ such that $\sum_{\ell=1}^{N} A_{\ell}^{*} A_{\ell} \leq I_{\mathcal{H}}$. Then for every polynomial $Q(z)$ with complex coefficients, we have

$$
\left\|Q\left(A_{1}, \ldots, A_{N}\right)\right\|_{\mathcal{H}} \leq\left\|Q\left(M_{1}, \ldots, M_{N}\right)\right\|_{\mathcal{A}} .
$$

The counterparts of the space $\mathcal{A}$ and of the operators $R_{\ell}$ have been recently introduced in the quaternionic setting [5,6], and the purpose of this article is to prove a von Neumann inequality in that setting. The lack of commutativity of the quaternions forces the choice of polynomials to be with real coefficients (Theorem 2.1).

\section{The quaternionic Drury-Arveson space and the statement of the main theorem}

We first briefly review the setting of hyperanalytic functions and the results of $[5,6]$. We denote by $\mathbb{H}$ the skew-field of real quaternions

$$
\mathbb{H}=\left\{x=x_{0}+x_{1} \mathbf{e}_{1}+x_{2} \mathbf{e}_{2}+x_{3} \mathbf{e}_{3}:\left(x_{0}, x_{1}, x_{2}, x_{3}\right) \in \mathbb{R}^{4}\right\},
$$

where the units $\mathbf{e}_{j}$ satisfy the Cayley multiplication table [6, Section 2.1]. We also let $\bar{x}=x_{0}-x_{1} \mathbf{e}_{1}-x_{2} \mathbf{e}_{2}-x_{3} \mathbf{e}_{3}$ and $|x|^{2}=x \bar{x}=\bar{x} x$. A function $f$ defined on an open set $\Omega \subset \mathbb{R}^{4}$ is called left-hyperanalytic (we will simply say hyperanalytic) if

$$
\frac{\partial}{\partial x_{0}} f+\mathbf{e}_{1} \frac{\partial}{\partial x_{1}} f+\mathbf{e}_{2} \frac{\partial}{\partial x_{2}} f+\mathbf{e}_{3} \frac{\partial}{\partial x_{3}} f=0
$$

holds in $\Omega$. We use the terms hyperanalytic and hyperholomophic interchangeably.

The quaternionic variable $x$ is not hyperanalytic, nor is, in general, the product of two hyperanalytic functions. The functions $\zeta_{\ell}(x)=x_{\ell}-\mathbf{e}_{\ell} x_{0}, \quad \ell=1,2,3$, are hyperanalytic and they form the building blocks of the hyperanalytic polynomials; note that they do not commute and that they are not independent variables in the sense that they all depend on $x_{0}$. For $\alpha=\left(\alpha_{1}, \alpha_{2}, \alpha_{3}\right) \in \mathbb{N}^{3}$, let

$$
\zeta^{\alpha}(x)=\zeta_{1}(x)^{\times \alpha_{1}} \times \zeta_{2}(x)^{\times \alpha_{2}} \times \zeta_{3}(x)^{\times \alpha_{3}},
$$


where the symmetrized product of $a_{1}, \ldots, a_{n} \in \mathbb{H}$ is defined by

$$
a_{1} \times a_{2} \times \cdots \times a_{n}=\frac{1}{n !} \sum_{\sigma \in S_{n}} a_{\sigma(1)} a_{\sigma(2)} \cdots a_{\sigma(n)},
$$

in which $S_{n}$ is the set of all permutations of the set $\{1, \ldots, n\}$ and where $\zeta_{1}(x)^{\times \alpha_{1}}$ means that the term $\zeta_{1}(x)$ appears $\alpha_{1}$ times among the $a_{j}$ in (2).

The Cauchy-Kovalevskaya product $\odot$ is an associative (but not commutative) product defined originally by Sommen in [7], which associates to two hyperanalytic functions another hyperanalytic function. It is different from the pointwise product which, in general, does not yield a hyperanalytic function, and is defined using the Cauchy-Kovalevskaya extension theorem. We will not review this aspect here, but mention that

$$
\left(\zeta^{\alpha} p\right) \odot\left(\zeta^{\beta} q\right)=\zeta^{\alpha+\beta} p q, \quad \alpha, \beta \in \mathbb{N}^{3}, \quad p, q \in \mathbb{M} .
$$

The quaternionic Drury-Arveson space is defined as the set

$$
\mathcal{A}_{\mathbb{\sharp}}=\left\{f(x)=\sum_{\alpha \in \mathbb{N}^{3}} \zeta^{\alpha}(x) f_{\alpha}: f_{\alpha} \in \mathbb{H},\|f\|_{\mathcal{A}_{\mathbb{H}}}^{2}:=\sum_{\alpha \in \mathbb{N}^{3}} \frac{\left|f_{\alpha}\right|^{2}}{\omega_{\alpha}}<\infty\right\}
$$

[5, Definition 1.1]. It is a right quaternionic Hilbert space (definitions are recalled in Section 3) with the inner product

$$
\langle f, g\rangle=\sum_{\alpha \in \mathbb{N}^{3}} \frac{1}{\omega_{\alpha}} \overline{g_{\alpha}} f_{\alpha} \text { with } g(x)=\sum_{\alpha \in \mathbb{N}^{3}} \zeta^{\alpha}(x) g_{\alpha} .
$$

Its elements are hyperanalytic in the ellipsoid

$$
\mathcal{E}=\left\{\left(x_{0}, x_{1}, x_{2}, x_{3}\right) \in \mathbb{R}^{4}: 3 x_{0}^{2}+x_{1}^{2}+x_{2}^{2}+x_{3}^{2}<1\right\} .
$$

It is the reproducing kernel (right quaternionic) Hilbert space with reproducing kernel

$$
k(x, y)=\left(1-\zeta_{1}(x) \overline{\zeta_{1}(y)}-\zeta_{2}(x) \overline{\zeta_{2}(y)}-\zeta_{3}(x){\overline{\zeta_{3}(y)}}^{-\odot}\right.
$$

where $-\odot$ denotes the inverse with respect to the Cauchy-Kovalevskaya product; see [6, Proposition 2.10]. This means that

$$
\langle f, k(\cdot, y) p\rangle_{\mathcal{A}_{\mathbb{H}}}=\bar{p} f(y), \quad p \in \mathbb{M}, \quad y \in \mathcal{E} .
$$

We need to introduce the backward shift operators on $\mathcal{A}_{\llbracket \llbracket}$. We set

$$
R_{\ell} f(x)=\sum_{\alpha \in \mathbb{N}^{3}} \zeta^{\alpha-e^{\ell}}(x) \frac{\alpha_{\ell}}{|\alpha|} f_{\alpha},
$$

where $e^{1}=(1,0,0), e^{2}=(0,1,0)$, and $e^{3}=(0,0,1)$. The operators $R_{\ell}$ are right linear and bounded on $\mathcal{A}_{\mathbb{\square}}$ and satisfy

$$
R_{1}^{*} R_{1}+R_{2}^{*} R_{2}+R_{3}^{*} R_{3}=I_{\mathcal{A}_{\nVdash}}-C^{*} C, \quad \text { where } \quad C f=f(0) .
$$


Moreover, their adjoints are the operators of the Cauchy-Kovalevskaya product by the $\zeta_{\ell}$, that is,

$$
R_{\ell}^{*} f=M_{\ell} f=\zeta_{\ell} \odot f=f \odot \zeta_{\ell} .
$$

The adjoint of a right linear operator $A$ on a right quaternionic Hilbert space $\mathcal{H}$ is the right linear operator $A^{*}$ satisfying

$$
\left\langle A h_{1}, h_{2}\right\rangle_{\mathcal{H}}=\left\langle h_{1}, A^{*} h_{2}\right\rangle_{\mathcal{H}}, \quad h_{1}, h_{2} \in \mathcal{H} .
$$

If $A=A^{*}, A$ is called self-adjoint.

We now quote our main theorem.

THEOREM 2.1 Let $\mathcal{H}$ be a right quaternionic Hilbert space and let $A_{1}, A_{2}, A_{3}$ be pairwise commuting right linear bounded operators on $\mathcal{H}$ such that

$$
A_{1}^{*} A_{1}+A_{2}^{*} A_{2}+A_{3}^{*} A_{3} \leq I_{\mathcal{H}} .
$$

Then for every hyperholomorphic polynomial $Q(x)$ with real coefficients, it holds that

$$
\left\|Q\left(A_{1}, A_{2}, A_{3}\right)\right\| \leq\left\|Q\left(M_{1}, M_{2}, M_{3}\right)\right\| .
$$

Let us make a number of comments on this result. The polynomials in the theorem have real coefficients because the Hilbert space $\mathcal{H}$ is a right Hilbert space. For $q \in \mathbb{H}$ and $T$ a right linear operator, the operator $q T$ makes sense only if $q$ is real, and the operator $T q$ is right linear only if $q$ is real.

In the proof of Theorem 2.1, we need the following result on square roots, which is well known and has a number of proofs in the case of complex Hilbert spaces. As in this latter case, the proof for the quaternionic case is based on the power series expansion of $\sqrt{1-z}$ in the open unit disk, and we omit it.

LEMma 2.2 Let T be a strictly contractive self-adjoint right linear operator on a right quaternionic Hilbert space $\mathcal{H}$. Then the operator

$$
D=I-\frac{1}{2} T+\frac{\frac{1}{2}\left(\frac{1}{2}-1\right)}{2 !} T^{2}-\frac{\frac{1}{2}\left(\frac{1}{2}-1\right)\left(\frac{1}{2}-2\right)}{3 !} T^{3}+\cdots
$$

is a self-adjoint contractive right linear operator on $\mathcal{H}$ satisfying $D^{2}=I-T$.

\section{Tensor products of quaternionic Hilbert spaces}

Tensor products of quaternionic Hilbert spaces do not seem to have been much studied (see [8,9] for some results). The difficulty is the noncommutativity of the quaternions. To make our point, let us go back to the basic definitions. Let $R$ be a ring. Recall that if $\mathcal{G}$ is a right module over $R$ and $\mathcal{H}$ is a left module over $R$, the tensor product $\mathcal{G} \otimes_{R} \mathcal{H}$ is merely a group [10, p. 208]. To get more structure, we need, for example, $\mathcal{H}$ to be a two-sided $R$-module. Then the following result holds ([10, Theorem 5.5(iii)] or [11, Section 3]). If $\mathcal{H}$ is a two-sided $R$-module and $\mathcal{G}$ is a right $R$-module, then the tensor product $\mathcal{G} \otimes \mathcal{H}$ is a right $R$-module. Moreover,

$$
(g \otimes h) r=(g \otimes h r) \quad \text { and } \quad(g p \otimes h)=(g \otimes p h), \quad h \in \mathcal{H}, g \in \mathcal{G}, r \in R .
$$


We refer to [12] for information and references on quaternionic Hilbert spaces. We recall the following definition (see, e.g. [12, Definition 5.5] and the references therein). A right quaternionic pre-Hilbert space $\mathcal{G}$ is a right vector space on $\mathbb{T}$ endowed with an $\mathbb{W}$-valued form $\langle\cdot, \cdot\rangle$ that has the following properties:

(1) it is Hermitian: $\langle f, g\rangle=\langle\bar{g}, f\rangle, \quad f, g \in \mathcal{G}$;

(2) it is positive: $\langle f, f\rangle \geq 0, \quad f \in \mathcal{G}$;

(3) it is nondegenenate: $\langle f, f\rangle=0 \Leftrightarrow f=0$;

(4) it is linear in the sense that $\langle f p, g q\rangle=\bar{q}\langle f, g\rangle p, \quad f, g \in \mathcal{G}, \quad p, q \in \mathbb{M}$.

The space $\mathcal{G}$ is a right quaternionic Hilbert space if it is complete with respect to the topology defined by the norm $\|f\|=\sqrt{\langle f, f\rangle}$.

Throughout this article, the notation $\mathcal{G} \otimes_{\mathbb{H}} \mathcal{H}$ is used for the topological tensor product of the quaternionic Hilbert spaces $\mathcal{G}$ and $\mathcal{H}$.

THEOREM 3.1 Let $\mathcal{H}$ be a separable two-sided quaternionic Hilbert space and let $\mathcal{G}$ be

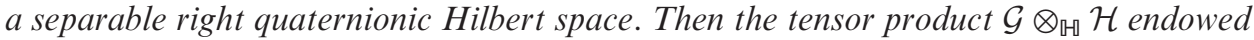
with the inner product

$$
\left\langle g_{1} \otimes h_{1}, g_{2} \otimes h_{2}\right\rangle_{\mathcal{G} \otimes_{\mathcal{H}} \mathcal{H}}=\left\langle\left(\left\langle g_{1}, g_{2}\right\rangle_{\mathcal{G}}\right) h_{1}, h_{2}\right\rangle_{\mathcal{H}}
$$

is a right quaternionic Hilbert space.

Proof Let $\left(h_{i}\right)$ (resp. $\left.\left(g_{i}\right)\right)$ be an orthonormal basis of $\mathcal{H}$ (resp. $\mathcal{G}$ ). Then (3) gives

$$
\left\langle g_{i_{1}} \otimes h_{j_{1}} p, g_{i_{2}} \otimes h_{j_{2}} q\right\rangle_{\mathcal{G} \otimes_{\mathcal{H}} \mathcal{H}}=\left\langle\left\langle g_{j_{1}}, g_{j_{2}}\right\rangle_{\mathcal{G}} h_{i_{1}} p, h_{i_{2}} q\right\rangle_{\mathcal{H}}=\left\{\begin{array}{cl}
\bar{q} p, & \text { if }\left(i_{1}, j_{1}\right)=\left(i_{2}, j_{2}\right) ; \\
0, & \text { otherwise. }
\end{array}\right.
$$

It follows that the right span of the elements of the form $g_{i} \otimes h_{j}$ endowed with the inner product (3) is a right quaternionic pre-Hilbert space. Then the set of elements of the form $\sum_{i, j} g_{i} \otimes f_{j} c_{i j}$, where the $c_{i j} \in \mathbb{H}$ are such that

$$
\sum_{i, j}\left|c_{i j}\right|^{2}<\infty
$$

is a right quaternionic Hilbert space.

\section{Proof of the main theorem}

We follow Drury's argument appropriately adapted to the quaternionic case. As in [1], it is convenient to work with the sequence space

$$
\ell_{2}\left(\mathbb{N}^{3}, \omega, \mathbb{H}\right)=\left\{\left(f_{\alpha}\right)_{\alpha \in \mathbb{N}^{3}}: f_{\alpha} \in \mathbb{H}, \sum_{\alpha \in \mathbb{N}^{3}} \omega_{\alpha}\left|f_{\alpha}\right|^{2}<\infty\right\}
$$

rather than $\mathcal{A}_{\mathbb{n}}$, where $\omega$ denotes the sequence $\left(\omega_{\alpha}\right)$. We endow $\ell_{2}\left(\mathbb{N}^{3}, \omega\right.$, $\left.\mathbb{H}\right)$ with the inner product

$$
\left\langle\left(f_{\alpha}\right),\left(g_{\alpha}\right)\right\rangle_{\ell_{2}\left(\mathbb{N}^{3}, \omega, \mathbb{H}\right)}=\sum_{\alpha \in \mathbb{N}^{3}} \omega_{\alpha} \overline{g_{\alpha}} f_{\alpha} .
$$


The operators

$$
\left(S_{\ell} f\right)_{\alpha}=f_{\alpha+e^{\ell}}, \quad \ell=1,2,3,
$$

are right linear and bounded on $\ell_{2}\left(\mathbb{N}^{3}, \omega\right.$, $\left.\mathbb{H}\right)$, they commute pairwise, and their adjoints are given by

$$
\left(S_{\ell}^{*} f\right)_{\alpha}=\frac{\alpha_{\ell}}{|\alpha|} f_{\alpha-e^{\ell}}, \quad \ell=1,2,3 .
$$

Moreover,

$$
S_{1}^{*} S_{1}+S_{2}^{*} S_{2}+S_{3}^{*} S_{3} \leq I_{\ell_{2}\left(\mathbb{N}^{3}, \omega, \mathbb{H}\right)} .
$$

These facts are proved as in the case of complex sequences and we omit their proofs here.

We first prove the theorem with $S_{1}, S_{2}, S_{3}$ in place of $M_{1}, M_{2}, M_{3}$. Let $A_{1}, A_{2}, A_{3}$ be as in the theorem. The operator

$$
I_{\mathcal{H}}-A_{1}^{*} A_{1}-A_{2}^{*} A_{2}-A_{3}^{*} A_{3}
$$

is positive. We first assume that

$$
r^{2} I_{\mathcal{H}}-A_{1}^{*} A_{1}-A_{2}^{*} A_{2}-A_{3}^{*} A_{3} \geq 0
$$

for some $r \in(0,1)$. The operator (4) is then strictly positive and we can apply Lemma 2.2 to define a positive operator $D$ such that

$$
D^{2}=I_{\mathcal{H}}-A_{1}^{*} A_{1}-A_{2}^{*} A_{2}-A_{3}^{*} A_{3} .
$$

We denote by $\widehat{\mathcal{H}}$ the Hilbert space $\mathcal{H}$ endowed with the norm

$$
\|h\|_{\widehat{\mathcal{H}}}=\|D h\|_{\mathcal{H}} \cdot
$$

Furthermore, we set

$$
\widetilde{\mathcal{H}}=\ell_{2}\left(\mathbb{N}^{3}, \omega, \widehat{\mathcal{H}}\right)
$$

this is similar to $\ell_{2}\left(\mathbb{N}^{3}, \omega, \mathbb{H}\right)$, but the terms of the sequences are members of $\widehat{\mathcal{H}}$, and the norm of $\widehat{\mathcal{H}}$ replaces the $|\cdot|$ of $\mathbb{H}$. Theorem 3.1 is used to prove the next auxiliary result, which of course is well-known in the complex case.

Proposition 4.1 The map

$$
\tau(h \otimes \xi)=(h \xi)
$$

is well defined and extends to a right linear unitary map between the right quaternionic Hilbert spaces $\widehat{\mathcal{H}} \otimes \ell_{2}\left(\mathbb{N}^{3}, \omega, \mathbb{H}\right)$ and $\widetilde{\mathcal{H}}$.

Proof Let $h \otimes \xi$ be an elementary tensor and let $q \in \mathbb{H}$. Then

$$
\tau((h \otimes \xi) q)=\tau(h \otimes(\xi q))=h \xi q=(h \xi) q=(\tau(h \otimes \xi)) q,
$$

and the right linearity of $\tau$ follows. 
For $k=1,2$, let $h_{k} \in \widehat{\mathcal{H}}$ and $\xi^{k}=\left(\xi_{\alpha}^{k}\right)$, where the sequences have only a finite number of nonzero entries. Then

$$
\begin{aligned}
\left\langle\tau\left(h_{1} \otimes \xi^{1}\right), \tau\left(h_{2} \otimes \xi^{2}\right)\right\rangle_{\widehat{\mathcal{H}} \otimes \ell_{2}\left(\mathbb{N}^{3}, \omega, \mathbb{H}\right)} & =\left\langle h_{1} \xi^{1}, h_{2} \xi^{2}\right\rangle_{\ell_{2}\left(\mathbb{N}^{3}, \omega, \widehat{\mathcal{H}}\right)} \\
& =\sum_{\alpha} \omega(\alpha)\left\langle h_{1} \xi_{\alpha}^{1}, h_{2} \xi_{\alpha}^{2}\right\rangle_{\widehat{\mathcal{H}}} \\
& =\sum_{\alpha} \omega(\alpha) \overline{\xi_{\alpha}^{2}}\left\langle h_{1}, h_{2}\right\rangle_{\widehat{\mathcal{H}}} \xi_{\alpha}^{1} \\
& =\left\langle\left\langle h_{1}, h_{2}\right\rangle_{\widehat{\mathcal{H}}^{-}} \xi^{1}, \xi^{2}\right\rangle_{\ell_{2}\left(\mathbb{N}^{3}, \omega, \mathbb{H}\right)} \\
& =\left\langle h_{1} \otimes \xi^{1}, h_{2} \otimes \xi^{2}\right\rangle \widehat{\mathcal{H} \otimes \ell_{2}\left(\mathbb{N}^{3}, \omega, \mathbb{H}\right)},
\end{aligned}
$$

and hence $\tau$ is an isometry. We now compute the adjoint of $\tau$. We denote by $\left(\widehat{h_{i}}\right)$ an orthonormal basis of $\widehat{\mathcal{H}}$. Let $H=\left(H_{\alpha}\right) \in \widetilde{\mathcal{H}}$. We prove that

$$
\tau^{*}(H)=\sum_{i} \widehat{h_{i}} \otimes\left(\left\langle H_{\alpha}, \widehat{h_{i}}\right\rangle_{\widehat{\mathcal{H}}}\right)_{\alpha}
$$

We have

$$
\begin{aligned}
\left\langle\tau^{*}(H), h \otimes \xi\right\rangle_{\widehat{\mathcal{H}} \otimes \ell_{2}\left(\mathbb{N}^{3}, \omega, \mathbb{H}\right)} & =\langle H, h \xi\rangle_{\ell_{2}\left(\mathbb{N}^{3}, \omega, \widehat{\mathcal{H}}\right)}=\sum_{\alpha} \omega(\alpha)\left\langle H_{\alpha}, h \xi_{\alpha}\right\rangle_{\widehat{\mathcal{H}}} \\
& =\sum_{\alpha}\left\langle\left(\left\langle H_{\alpha}, h\right\rangle_{\widehat{\mathcal{H}}}\right)_{\alpha}, \xi\right\rangle_{\ell_{2}\left(\mathbb{N}^{3}, \omega, \mathbb{H}\right)}
\end{aligned}
$$

on the one hand, and

$$
\begin{aligned}
\left\langle\sum_{i} \widehat{h_{i}} \otimes\left(\left\langle H_{\alpha}, \widehat{h_{i}}\right\rangle_{\widehat{\mathcal{H}}}\right)_{\alpha}, h \otimes \xi\right\rangle_{\widehat{\mathcal{H}} \otimes \ell_{2}\left(\mathbb{N}^{3}, \omega, \mathbb{W}\right)} & =\sum_{i}\left\langle\left(\left\langle H_{\alpha}, \widehat{h_{i}}\right\rangle_{\widehat{\mathcal{H}}}\left\langle\widehat{h}_{i}, h\right\rangle_{\widehat{\mathcal{H}}}\right)_{\alpha}, \xi\right\rangle_{\ell_{2}\left(\mathbb{N}^{3}, \omega, \mathbb{H}\right)} \\
& =\left\langle\left(\left\langle H_{\alpha}, h\right\rangle_{\widehat{\mathcal{H}}}\right)_{\alpha}, \xi\right\rangle_{\ell_{2}\left(\mathbb{N}^{3}, \omega, \mathbb{H}\right)}
\end{aligned}
$$

on the other hand, and the claim on the adjoint follows.

We can now prove that $\tau$ is unitary.

$$
\tau \tau^{*}(H)=\tau\left(\sum_{i} \widehat{h}_{i} \otimes\left(\left\langle H_{\alpha}, \widehat{h}_{i}\right\rangle_{\widehat{\mathcal{H}}}\right)_{\alpha}\right)=\sum_{i} \widehat{h}_{i}\left(\left\langle H_{\alpha}, \widehat{h}_{i}\right\rangle_{\widehat{\mathcal{H}}}\right)_{\alpha}=\left(H_{\alpha}\right)
$$

and

$$
\begin{aligned}
\tau^{*} \tau(h \otimes \xi) & =\tau^{*}(h \xi)=\sum_{i} \widehat{h}_{i} \otimes\left(\left\langle(h \xi)_{\alpha}, \widehat{h}_{i}\right\rangle_{\widehat{\mathcal{H}}}\right) \\
& =\sum_{i} \widehat{h_{i}} \otimes\left\langle h, \widehat{h}_{i}\right\rangle_{\widehat{\mathcal{H}}} \xi=\left(\sum_{i} \widehat{h_{i}}\left\langle h, \widehat{h_{i}}\right\rangle_{\widehat{\mathcal{H}}}\right) \otimes \xi=h \otimes \xi,
\end{aligned}
$$

since $(h \otimes p \xi)=(h p) \otimes \xi$.

We now conclude the proof of the theorem in a number of steps. The proof of Step 1 is the same as in the complex case and will be omitted. 
Step 1: The map $\Theta$ defined by

is an isometry from $\mathcal{H}$ into $\ell_{2}\left(\mathbb{N}^{3}, \omega, \widehat{\mathcal{H}}\right)$.

$$
(\Theta(h))_{\alpha}=A^{\alpha} h, \quad h \in \mathcal{H},
$$

We define two other kinds of shifts. The map $S^{\mu}$ carries a sequence $\left(h_{\alpha}\right) \in \widehat{\mathcal{H}}$ to $\left(h_{\alpha+\mu}\right)$. The map $\widetilde{S}^{\mu}$ has the same action on a sequence $\left(H_{\alpha}\right) \in \widetilde{\mathcal{H}}$.

Step 2: For $\mu \in \mathbb{N}^{3}$, it holds that $\widetilde{S}^{\mu} \tau=\tau\left(I_{\widehat{\mathcal{H}}} \otimes S^{\mu}\right)$.

Indeed, let $h \otimes\left(h_{\alpha}\right)$ be an elementary tensor in $\widehat{\mathcal{H}} \otimes \ell_{2}\left(\mathbb{N}^{3}, \omega, \mathbb{H}\right)$. Then

$$
\begin{aligned}
\widetilde{S}^{\mu} \tau\left(h \otimes\left(h_{\alpha}\right)\right) & =\widetilde{S}^{\mu}\left(h h_{\alpha}\right)=\left(h h_{\alpha+\mu}\right)=\tau\left(\left(h \otimes h_{\alpha+\mu}\right)\right)=\tau\left(h \otimes S^{\mu}\left(h_{\alpha}\right)\right) \\
& =\left(\tau\left(I_{\widehat{\mathcal{H}}} \otimes S^{\mu}\right)\right)\left(h \otimes\left(h_{\alpha}\right)\right) .
\end{aligned}
$$

Step 3: For $\mu \in \mathbb{N}^{3}$, it holds that $\widetilde{S}^{\mu} \Theta=\Theta A^{\mu}$, and hence

$$
\left(I_{\widehat{\mathcal{H}}} \otimes S^{\mu}\right)\left(\tau^{*} \Theta\right)=\left(\tau^{*} \Theta\right) A^{\mu} \quad \text { and } \quad\left(I_{\widehat{\mathcal{H}}} \otimes Q(S)\right)\left(\tau^{*} \Theta\right)=\left(\tau^{*} \Theta\right) Q(A),
$$

where $Q\left(x_{1}, x_{2}, x_{3}\right)=\sum_{\mu} x^{\mu} q_{\mu}$ is a polynomial with real coefficients.

Indeed, for $h \in \widehat{\mathcal{H}}$, we have

$$
\begin{aligned}
\tau\left(I_{\widehat{\mathcal{H}}} \otimes S^{\mu}\right) \tau^{*} \Theta h & =\tau\left(I_{\widehat{\mathcal{H}}} \otimes S^{\mu}\right) \tau^{*}\left(A^{\alpha} h\right) \\
& =\tau\left(I_{\widehat{\mathcal{H}}} \otimes S^{\mu}\right)\left(\sum_{i} \widehat{h_{i}} \otimes\left(\left\langle A^{\alpha} h, \widehat{h_{i}}\right\rangle_{\widehat{\mathcal{H}}}\right)_{\alpha}\right) \\
& =\tau\left(\sum_{i} \widehat{h_{i}} \otimes\left(\left\langle A^{\alpha+\mu} h, \widehat{h_{i}}\right\rangle_{\widehat{\mathcal{H}}}\right)_{\alpha}\right) \\
& =\sum_{i} \widehat{h_{i}}\left(\left\langle A^{\alpha+\mu} h, \widehat{h_{i}}\right\rangle_{\widehat{\mathcal{H}}}\right)_{\alpha}=\left(A^{\alpha+\mu} h\right) .
\end{aligned}
$$

Then the result also holds for any finite linear combination $\sum_{\mu} x^{\mu} q_{\mu}$ with real $q_{\mu}$.

The proof of the next step is a direct consequence of (5).

Step 4: von Neumann inequality holds with the operators $S_{1}, S_{2}, S_{3}$.

Step 5: von Neumann inequality holds with the operators $M_{1}, M_{2}, M_{3}$.

Indeed the map $T$ defined by

$$
T\left(f_{\alpha}\right)=\sum_{\alpha \in \mathbb{N}^{3}} \zeta^{\alpha}(x) f_{\alpha} \omega(\alpha)
$$

is an isomorphism of right quaternionic Hilbert spaces from $\ell_{2}\left(\mathbb{N}^{3}, \omega, \mathbb{H}\right)$ onto $\mathcal{A}_{\mathbb{W}}$, and its adjoint is given by

$$
T^{*} f=\left(\frac{f_{\alpha}}{\omega_{\alpha}}\right)_{\alpha} \text { with } f(x)=\sum_{\alpha} \zeta^{\alpha} f_{\alpha} .
$$

Further,

$$
\begin{aligned}
\left(T S_{\ell} T^{*}\right)(f) & =T S_{\ell}\left(\frac{f_{\alpha}}{\omega_{\alpha}}\right)=T\left(\left(\frac{f_{\alpha+e^{\ell}}}{w_{\alpha+e^{\ell}}}\right)\right)=\sum_{\alpha} \zeta^{\alpha} \frac{f_{\alpha+e^{\ell}}}{\omega_{\alpha+e^{\ell}}} \omega_{\alpha} \\
& =\sum_{\alpha} \zeta^{\alpha} f_{\alpha+e^{\ell}} \frac{\alpha_{\ell}+1}{|\alpha|+1}=\sum_{\alpha \geq e^{\ell}} \zeta^{\alpha-e^{\ell}}(x) f_{\alpha} \frac{\alpha_{\ell}}{|\alpha|}
\end{aligned}
$$


and

$$
\begin{aligned}
\left(T S_{\ell}^{*} T^{*}\right)(f) & =T S_{\ell}^{*}\left(\frac{f_{\alpha}}{\omega_{\alpha}}\right)=T\left(\frac{f_{\alpha-e^{\ell}}}{\omega_{\alpha-e^{\ell}}} \frac{\alpha_{\ell}}{|\alpha|}\right)=\sum_{\alpha \geq e^{\ell}} \zeta^{\alpha}(x) \frac{f_{\alpha-e^{\ell}}}{\omega_{\alpha-e^{\ell}}} \frac{\alpha_{\ell}}{|\alpha|} \\
& =\sum_{\alpha \geq e^{\ell}} \zeta^{\alpha}(x) f_{\alpha-e^{\ell}} \frac{\alpha_{\ell}}{|\alpha|} \frac{\left(\alpha-e^{\ell}\right) !}{(|\alpha|-1) !}=\zeta_{\ell} \odot f=M_{\ell} f .
\end{aligned}
$$

Thus

$$
\begin{aligned}
\left\|Q\left(S_{1}, S_{2}, S_{3}\right)\right\| & =\left\|Q\left(T\left(S_{1}, S_{2}, S_{3}\right) T^{*}\right)\right\|=\left\|Q\left(T\left(S_{1}, S_{2}, S_{3}\right) T^{*}\right)^{*}\right\| \\
& =\left\|Q\left(T\left(S_{1}^{*}, S_{2}^{*}, S_{3}^{*}\right) T^{*}\right)\right\|=\left\|Q\left(M_{1}, M_{2}, M_{3}\right)\right\|,
\end{aligned}
$$

where the next to last equality uses the fact that $Q$ has real coefficients, and the last equality uses (6).

It remains to let $r \rightarrow 1$ to conclude the proof.

\section{References}

[1] S.W. Drury, A generalization of von Neumann's inequality to the complex ball, Proc. Amer. Math. Soc. 68 (1978), pp. 300-304.

[2] W. Arveson, Subalgebras of $C^{*}$-algebras III: Multivariable operator theory, Acta Math. 181 (1998), pp. 159-228.

[3] G. Popescu, von Neumann Inequality for $\left(B(\mathcal{H})^{n}\right)_{1}$, Math. Scand. 68 (1991), pp. 292-304.

[4] D. Alpay and H.T. Kaptanoğlu, Some finite-dimensional backward-shift-invariant subspaces in the ball and a related interpolation problem, Integral Eqns. Oper. Theory 42 (2002), pp. 1-21.

[5] D. Alpay, M. Shapiro, and D. Volok, Espaces de de Branges Rovnyak et fonctions de Schur: Le cas hyper-analytique, C. R. Math. Acad. Sci. Paris 338 (2004), pp. 437-442.

[6] D. Alpay, M. Shapiro, and D. Volok, Rational hyperholomorphic functions in $\boldsymbol{R}^{4}$, J. Funct. Anal. 221 (2005), pp. 122-149.

[7] F. Sommen, A product and an exponential function in hypercomplex function theory, Appl. Anal. 12 (1981), pp. 13-26.

[8] L.P. Horwitz and A. Razon, Tensor product of quaternion Hilbert modules, in Classical and Quantum Systems, H.-D. Doebner, W. Scherer, and F. Schroeck, eds., World Scientific, River Edge, 1993, pp. 266-268.

[9] A. Razon and L.P. Horwitz, Uniqueness of the scalar product in the tensor product of quaternion Hilbert modules, J. Math. Phys. 33 (1992), pp. 3098-3104.

[10] T.W. Hungerford, Algebra, Holt, Rinehart and Winston, New York, 1974.

[11] N. Bourbaki, Éléments de Mathématique: Algèbre, Chapitres 1 à 3, Hermann, Paris, 1970.

[12] D. Alpay and M. Shapiro, Reproducing kernel quaternionic Pontryagin spaces, Integral Eqns. Oper. Theory 50 (2004), pp. 431-476. 